\title{
Rebuilding growth mechanisms through visual observations
}

\author{
Maria Raimo $^{1}$ Elvira Lotti ${ }^{2}$
}

Received: 31 March 2016/Accepted: 27 May 2016/Published online: 11 June 2016

(C) Springer International Publishing Switzerland 2016

\begin{abstract}
This article conveys useful key factors to rebuilt the solidification of polycrystalline materials, as well as the growth history of organisms and their parts, and to establish the metrics of the growth. The microscopic analysis of solids is founded on the classical nucleation theory and on interconnections between the heat flow pathway and geometry of grains and interfaces. Particular order of the grains, such as alignment, indicates non-isotropic heat transport during solidification, for instance, due to the presence of extraneous filamentous material or to heat exchange by convection along definite directions. Morphological analysis is also shown to be valuable to detect particular thermal effects caused by changes of environmental factors during growth. Relatively to solids, the growth pattern of living bodies is complicated by mass transfer between cells and environment. However, several similarities with non-living matters can be often found.
\end{abstract}

Keywords Growth · Polymer solidification · Rhythmicity · Biomimicry $\cdot$ Fungi

Maria Raimo

maria.raimo@cnr.it

1 Consiglio Nazionale delle Ricerche, Istituto per i Polimeri, Compositi e Biomateriali, Via Campi Flegrei, 34, 80078 Pozzuoli (NA), Italy

2 Consiglio per la Ricerca e la Sperimentazione in Agricoltura, Centro di ricerca per la patologia vegetale, Via C.G. Bertero, 22, 00156 Rome, Italy

\section{Introduction}

The simple microscopic observation of solids, in the wider sense of the term, can reveal amazing structures and tell us considerably about the surrounding conditions during their formation, provided that the appropriate reading keys are accessible. For instance, the growth pattern of the skeleton in living organisms may record physiological and environmental changes [1] which remain imprinted in the final structure.

Growth is a general process that applies either to phase transitions and biological processes in living organisms as bacteria, animals, plants, and parts of them. Decoding the morphological information contained in a skeleton, as well as in crystals of synthetic or natural materials, is of great importance to rebuilt the modality of the whole growth at the end of the process, especially when direct observations cannot be made in the intervening period. Investigations on growth can be very useful not only in theory but also in practice, to prevent noxious effects as those related to the development of pathogen microorganisms or to undesired precipitation of solid substances. It is surprising that the history of a solid, and therefore, the variations occurred during its growth, can be often rapidly deduced by a simple look under an optical microscope, without complex instrumental examinations. Indeed, structural changes found in naturally occurring materials, such as minerals and ice, as well as disparities in the size of annual rings of trees, are either very useful to discover climate and ambient variations occurred in the past. Direct analogues to tree rings are coral and shell bands, since the annual growth increments of these marine organisms provide a quantitative indication of their age and water temperature variability [2]. Structural and morphological analysis can also support the results of 
sophisticated analytical techniques, as those based on isotopic composition, for dating of ancient finds. Variation of conditions during the solidification of non-natural materials, especially polymers, also causes various effects detectable on different size scales. Indeed, distortions or modifications of the elemental cell of a crystal lattice on nanometres scale, with the appearance of polymorphic forms, are often revealed by X-ray diffraction. Changes in morphology and size of crystals are also expected, on the length scale of micrometres, as consequence of experimental changes in solidification procedures and equipment. For instance, temperature spikes during polymer solidification are evidenced by the presence of rings within spherulites, which are the basic morphological units of polycrystalline samples observable through a polarizing microscope. Usually, polymer spherulites grown at a constant temperature are not banded. If a temperature change occurs occasionally, a black ring within spherulites will separate an external circular corona from an inner circle grown at a different temperature. Intermittent temperature changes produce concentring rings within each spherulite and, depending on the exact temperature variation with time, the distance between two successive rings may be constant or variable [3]. Polymer spherulites, indeed, are often banded, especially when developed under temperature jumps. A few authors have found that the radial growth rate of even isothermally grown banded spherulites is unequal within two successive bands, since an interchange between slow and fast rate during crystallization allows the progress of crystallization by rhythmic heat dissipation $[4,5]$. It is worth observing that similar banded growth patterns occur frequently in nature as consequence of temperature or concentration gradients. Banding as symptom of rhythmic growth is, for instance, found in asexual spore production of fungi, such as Neurospora crassa. As the mycelium grows along the surface of a solid medium it begins, after several hours, to form aerial hyphae at the growing front. This aerial growth continues for several hours and then stops, allowing surface growth to be restored and continued for a period of time; afterwards, aerial hyphae are again formed and so on [6]. The macroscopic effect of this dual growth is the observation on solid agar media of a series of conidiating bands (asexual spore formation) that develop c.a. $22 \mathrm{~h}$ apart, alternating with areas of thin filamentous growth referred to as "interbands", either in Petri plates or long glass tubes (race tubes). Neurospora crassa shows a periodicity of growth and reproduction with a period of almost $22 \mathrm{~h}$ in the temperature range of 18-30 ${ }^{\circ} \mathrm{C}$ [7]. Since Neurospora is considered to grow on a surface medium at a constant linear rate, the period of the rhythm can be calculated from the distance between consecutive bands, whereas the phase of the rhythm is determined from the position of the bands relative to the growth fronts [7]. Although most of the first reports on the formation of zones in developing fungal colonies indicate that the zones are caused by a repetitive oscillation of an environmental factor, several studies have also evidenced an endogenous origin of the periodic growth in $\mathrm{Neu}$ rospora crassa. Rhythmic growth behaviors observed in biological systems are in principle more complicate than banding in polymers, where the only oscillating parameter is generally the temperature. Living systems, indeed, exchange mass and heat through metabolic pathways, which are networks of chemical reactions mostly catalyzed by enzymes. Temperature oscillations affect either the kinetics or thermodynamics of reactions, and vice versa, the heat accompanying reactions may produce local temperature fluctuations. Indeed, temperature is the simpler factor controlling chemico-physical processes. As a consequence, even when biological rhythms are controlled by oscillating concentrations of metabolites, they are not entirely temperature independent [6]. For instance, when the temperature of a strain of Neurospora grown on a definite medium in a race tube for 19 days is increased from 15 to $25{ }^{\circ} \mathrm{C}$, a frequency of bands associated with conidiation of almost three times the value obtained at temperature $10{ }^{\circ} \mathrm{C}$ lower is produced, although the linear rate of the mould down the race tube is not significantly affected [6]. Rhythmic processes in both living and nonliving systems, despite remarkable differences, express the response of a steady state to chemical or physical perturbations. For instance, intermittent slowdown of the growth during polymer crystallization $[3,5,8]$ is the most natural countermove to local superheating effects due to either heat release at the growth front and slow heat transfer by conduction. The heat of solidification developed at the growth front is hardly removed by conduction in low thermal conductivity substances as polymers, and the progress of liquid-solid transitions at low undercooling may undergo rhythmic temporary stops. During solidification of metals, a similar counteraction towards superheating can be observed by naked eye as increase in brightness according to a phenomenon called recalescence. Indeed, a wave or periodic response of a process gives the possibility to breakdown the resistance of surroundings to the process itself. In biological systems, rhythmic patterns as that adopted by Neurospora crassa clearly reveal adaptation to environmental changes, either of exogenous or endogenous (i.e., thermal, chemical, or genetic) origin [9]. In all cases, rhythmic behaviors allow to overcome temporary kinetic or thermodynamic hindrance due to effects, as the accumulation of reaction products or heat, originated during a process. Temperature increases within physiological intervals cause generally faster growth of living bodies as trees, as well as 
major fungal reproduction, since generally high temperatures accelerate chemical reactions. A physical process as solidification instead slows down as consequence of temperature enhancements, since it is exothermal.

As similarities, also differences between the growth patterns of living and non-living bodies are useful to deduce historical events during growth. In some organisms, the growth can be restricted to special regions, such as at tips in fungi or near the apex of plant shoots [10]. Mycelial fungi, being non-cellular according to the most general meaning of the term, may increase in volume, numbers of nuclei, and amount of cytoplasm, but cannot enhance in the number of cells [11]. However, the main difference between most of living and non-living bodies consists generally in the way of accommodation of new cells or fresh material: with a few exceptions, biological organisms grow mainly by internal increase of cells number and size, whereas the growth of non-living matter entails the addition of new material only to the exterior edges. For instance, polycrystalline solids, such as polymers, show a tessellated structure resulting from coalescence of several circular "crystals" called spherulites, originated from liquids by a mechanism of nucleation and external growth [12], whereas the growth of biological systems generally entails interior growth and division of pre-existent cells in already compact tissues. This peculiar growth, besides the flexibility of the cellular membranes and the presence of liquid cytoplasm inside cells, makes structures and properties of non-living materials very different from that of biological tissues. In spite of these differences, nature may magisterially harmonize the coexisting growth mechanisms of complex biological tissues and simple inorganic compounds. An example of concurrent growths is the enlargement of a shell to re-accommodate a body. Meanwhile, the growth of the internal organism occurs by the enhancement of the cells number, and the size of the shell increases by adding material (mostly calcium carbonate) to the margins. In several gastropods and cephalopods, such as nautilus, the enlargement of shells occurs by an underlying spiral growth, a pathway that allows to increase the size by adding materials to one end without any change in the shape. Interestingly, spiral growth has also been observed in the polymer crystallization [13] and in the portion of plants, where growth occurs by delivery of primordia to tips [10]. For instance, the seeds produced in the centre of sunflowers and then moved towards the boundary show the most efficient way of filling space by spiral patterns. This spiral growth is likely originated by space restrictions and "steric repulsion" between contiguous seeds, since each seed is shifted at a certain angle in relation of the successive seed [10]. The angle depends on the radial velocity, the distance of the seed from the centre, and the period of the growth. The golden angle (c.a. $137.5^{\circ}$ ), which arises from the ratio between two subsequent Fibonacci numbers [14], allows to optimize the space taken by seeds, maximising the number of seeds per surface unit.

\section{Theoretical background}

The study of the relationship between body size and shape as a function of time [15] is a traditional branch of biology, since it is very important for the environmental adaptation and evolution of organisms. When the growth of different parts, or organs, of an individual organism occurs with equal rates, so that the ratio between their sizes is constant throughout the considered lifetime, the growth is defined isometric and the organism preserves its shape. The growth of animals is typically allometric, although isometric growth may also be observed, as in locusts and fishes. As a consequence of isometric growth, changes in size are not accompanied by changes in shape or external form [16]. The characteristic of living bodies, relatively to crystals, is the continuous use and transformation of external materials, or food, for growth. The development of living matters entails, therefore, a net increase of weight during growth. On the contrary, crystals grow only at expenses of the liquid phase (the equivalent of "food" for biosystems) in which they are immersed and, therefore, if the liquid is not continuously added, the total weight of the solid-liquid system remains constant; meanwhile, only the solid phase increases. This is why the growth of biological system is generally expresses by the mass, whereas for crystals are more conveniently used variable quantities proportional to the mass, such as the linear extension, the volume, or the solidification heat [12]. As shown in the next paragraphs, under specific conditions, also the growth of filamentous fungi may be expressed by following the length of hyphae, thus avoiding the need of extraction of the mycelium from the culture medium.

Since the growth may be defined in various ways, trustworthy comparisons between growth rates of similar matters may be only based on data attained from equivalent measuring methods.

\section{Metrics of growth}

\section{Polymer crystallization}

Direct observations on polymers crystallizing from melt mainly reveal the gradual growth of circular crystalline units usually with micrometre dimensions, referred to as spherulites. The original crystalline embryos producing spherulites fall in the length scale of nanometres and, 
therefore, are not observable by optical microscopy. According to the classical nucleation theory, crystalline nuclei are considered to be shaped as geometrical figures with the lowest surface energy that is circles or spheres. Since temperature controls liquid-solid transitions and crystallization is exothermal, the growth rate depends on the rate of heat removal from the solid front and increases with decreasing temperature [17]. The development of circular crystalline units throughout crystallization is only possible if the growth occurs, relatively to heat transfer, in isotropic media [18]. Indeed, an extending circle can continuously keep its shape only if the linear growth rates along all the radial directions are equal; otherwise, a facet crystal should arise [19]. The assumption of rounded geometry of the nuclei is justified by the capability of circles and spheres to ensure the minimum surface tension. Surfaces have generally a high energy, since atoms on the boundary cannot entirely satisfy their tendency to be wholly incorporated in the solid phase. As shown in Fig. 1, the formation of highly dispersed particles with a large surface area is not convenient, whereas the minimization of the particles surface, relatively to the whole mass or volume of the new phase, is associated to a lower energy cost. Indeed, only a few nuclei achieve a critical size to subsequently grow, whereas smaller nuclei re-dissolve, so that the increase in free energy due to the creation of surfaces does not exceed the free energy decrease caused by the formation of solid bulk. Since crystallization is exothermal and the growth rate depends on the rate of heat removal from the solid front, the development of circular crystalline units indicates that the rate of heat transfer does not depend on the particular direction, i.e., heat propagation is isotropic.

The instantaneous true growth rate of bi-dimensional crystals (i.e., the mass of the solid formed in a time unit) is not readily measurable [12]. The direct measurement of the mass of the solid produced at various times during crystallization would entail, beforehand, the recurrent stop of crystallization and the solid-liquid separation. This is practically very difficult to achieve or even unattainable. On the other hand, the rigorous estimation of the growth rate through visual observations would require the knowledge of the number of spherulites and their specific dimensions instant by instant. Fortunately, the development of the solid phase at a nominally constant temperature can be followed by simply measuring the advancement of each side of facet crystals or, for circularly developing crystals, the length of spherulites radius [19]. We will see in the next paragraphs that the length of the radius of spherulites can also be conveniently used, instead of the mass or other proportional quantities, to study the metrics of polycrystalline growth. Figure 2 shows, for instance, that the growth of two spherulites with the same linear growth rate is not isometric, unless spherulites nucleate simultaneously. Indicating with $r$ and $\delta$, respectively, the radius of a spherulite at a specific time $t$ and the mass of a surface unit of polymer, the total mass $m$ of $n$ simultaneously growing spherulites can be written as function of the area $A(t)$ of each spherulite by the formula: $m=n \delta A=n \delta \pi r^{2}$. By passing to differentials: $\mathrm{d} m=$ $2 n \delta \pi r \mathrm{~d} r$, that is: $\mathrm{d} m / \mathrm{d} t=2 n \delta \pi r \mathrm{~d} r / \mathrm{d} t$. In other words, assuming simultaneous nucleation and considering a spherulite annulus with infinitesimal thickness and mass (i.e., the annulus with radii $r$ and $r+\mathrm{d} r$, and mass $=\mathrm{d} m$ ), the instantaneous growth rate $\mathrm{d} m / \mathrm{d} t$ of a spherulitic sample can be obtained as a function of $r$, and hence of $t$, multiplying the number of spherulites $n$ by the mass of a spherulite surface unit $\delta$ and by the length $2 \pi r$ of the inner circumference of the annulus, and the product again by the so-called linear growth rate $\mathrm{d} r / \mathrm{d} t$ (which represents the rate of increase of the radius $r$ of a spherulite and is generally constant and uniform in space). To determine an average value of the true growth rate in a definite time interval, the mass of a circular corona with finite thickness

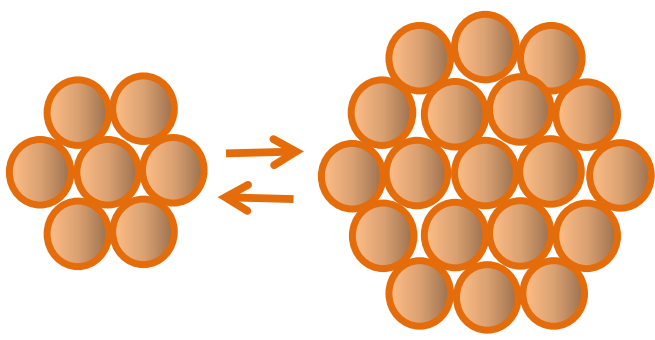

Fig. 1 Growth of an almost rounded nucleus with an initial subcritical dimension. In the first stage, the growth is not effective since too small nuclei may re-dissolve in the melt. Only when the

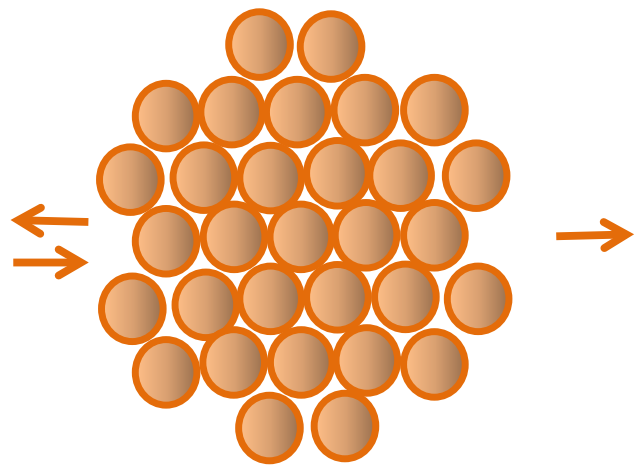

number of internal atoms overcomes the number of superficial atoms a crystal will be developed 


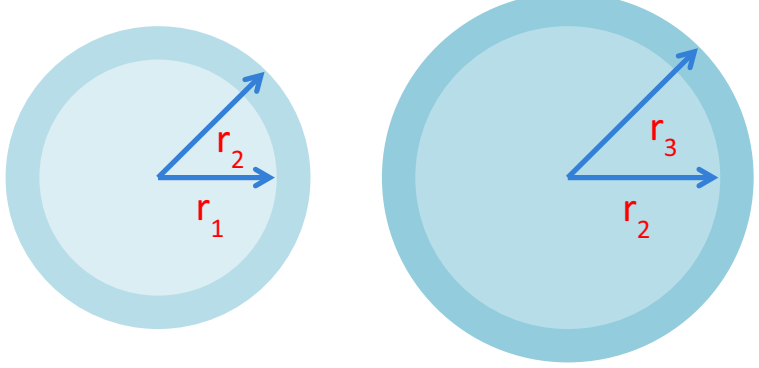

Fig. 2 Two spherulites growing with the same linear growth rate function $G(t)$, but nucleated at two different times. Indicating with $r_{1}$ and $r_{2}$, the respective radius at a time $t$, and with $r_{2}$ and $r_{3}$, the radii at a time $t^{\prime}$, it is evident that $r_{2}=r_{1}+\Delta r$ and $r_{3}=r_{2}+\Delta r$. Therefore, if $r_{1} \neq r_{2}$, it must also be: $r_{2} / r_{1} \neq r_{3} / r_{2}$ and the growth cannot be isometric

$\Delta m$ has to be considered. This mass can be obtained, for planar spherulites, as the product of the mass of polymer per surface unit and the increment of area of the spherulites in the considered time intervals. Indicating with $r$ and $R$, the radius of the smaller and bigger circumferences defining an annulus, the area of the annulus $\Delta A$, is given by $\pi\left(R^{2}-r^{2}\right)$ and, hence: $\Delta m=\delta \pi\left(R^{2}-r^{2}\right)$. For instance, the area and the mass of an annulus with unitary thickness result, respectively, $\pi(2 r+1)$ and $\delta \pi(2 r+1)$, since $R=r+1$.

The function $r(t)$ can be obtained by photographing at definite times a crystallizing specimen under a microscope and measuring the radius of selected spherulites. When the function $r(t)$ is linear, also the true growth rate $\mathrm{d} m / \mathrm{d} t$ increases proportionally with time, since the derivative $g=$ $\mathrm{d} r / \mathrm{d} t$ of the experimental function $r(t)$ is constant and can be easily calculated as the slope of the $r-t$ straight line. Different from the true growth rate [12], the linear growth rate of bi-dimensional crystals is indeed generally found to be time independent (i.e., constant), and a linear trend of the spherulites radius as a function of the time is experimentally observed. It is worth observing that also the growth kinetics of microbial colonies and filamentous fungi may be conveniently followed by measuring the radial expansion or hyphal elongation in the conventional Petri dish cultures [20] and often a constant radial growth rate is also found [21, 22]. Beyond hyphal elongation at the tips, the growth of fibrous fungi also occurs by branching; polymer spherulites often grow with a similar splitting mechanism of radiating lamellae [23]. It should, however, be observed that tips of fibrillar crystals grow exclusively at expense of external liquid materials, whereas the extension of leading hyphae and secondary branches in fungi seems to entail a short internal diffusion of "material" from few segments (septa) behind the tips, before the building of new portions of mycelium is done at the tips. Indeed, fungal segments behind the tips do not extend, even if they can be considered to contribute to the construction of new hyphal walls by allowing the internal passage of "food" to the tips. However, the assimilation and utilization of food for new wall construction seem to be only confined to the surrounding area of the fungal tips. Therefore, morphological and metric analogies between the growth of spherulites and mycelia are due to the fact that, ultimately, both systems incorporate external material or food directly at their growth fronts. It is also likely that branching of hyphae occurs in correspondence of segments, where the food concentration is locally higher [11].

The formation mechanisms of crystals and natural organisms show several other analogies. Polymer spherulites may be produced not only by two early recognised mechanisms (namely by radial extension of fibrous crystals developing lateral branches, in turn able to branch, and by sheaf-like mechanisms, widely described in the literature $[24,25])$, but also according to spiralizing patterns. Spiral growth is very common in nature and appears in shells [26], in a huge number of crystals [27] and even in galaxies. The addition of new material to crystalline nuclei in a spiraling way, depending on the type and the pitch of the spiral, may lead to a growth front almost indistinguishable from a circumference. Because boundaries of spirals with a low pitch may be at first sight indistinguishable from circles, only very few authors have recognised polymer spherulites spirally grown [28]. Indeed, the spiral growth mechanism can be clearly identified by optical or electronic microscopy only when spherulites show inner rings or bands and their centres are evidently shaped as a single or double spiral [29]. A single spiral spherulite is shown, for example, in Fig. 3.

Isotropic growth of spherulites may be perturbed in the presence of anisotropic materials, such as fibers, in the melt. Substances with one dimension much longer than the

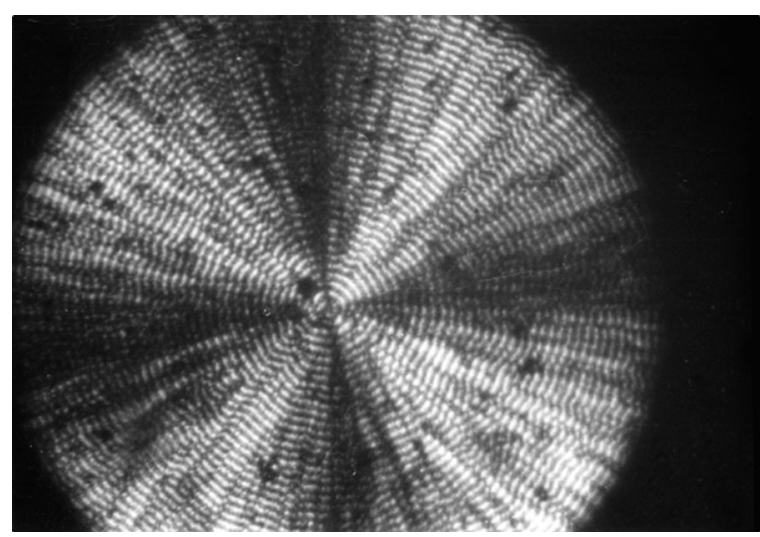

Fig. 3 Micrograph of a banded spherulite of polyhydroxybutyrate developed from a liquid blend. Although the spherulite boundary is hardly distinguishable from a circle, it is possible to identify a spiral growth pattern looking at the center of the spherulite 
other two are anisotropic not only relatively to the shape but also with respect to physical properties and generally show very different thermal conductivities along various directions [30]. Indeed, fibers are generally able to conduct heat along their axis much faster than isotropic media [31]. Therefore, when fibers are immersed in polymer melts, premature nucleation of embryos occurs along their axis and the crowding of spherulites causes fast coalescence with lack of circular shape, forcing the original spherulites to grow further only transversally to the fiber. The easier nucleation process along a fiber axis reflects the common ability of fibers to dissipate heat faster than polymer melts [32]. In other words, in the vicinity of a fiber local temperature decreases are more probable and favour the generation of embryos of solid phase with sufficient size to grow. Spherulites with the center laying on a fiber axis soon collide one another, then they can develop only transversally to the fiber, finally colliding with late spherulites nucleated in the melt. These latter have linear dimensions equal or smaller than the transversal dimensions of spherulites nucleated on the fibers. Therefore, as shown in Fig. 4, the presence of randomly arranged spherulites smaller than the maximum transversal dimension of spherulites laying out on a fiber is an evidence of the high heat diffusivity along fibers [32].

Before to go into the metrics of growth of solids, we can have a look at real structures of polymers. For instance, in Fig. 5, the tessellated structure of the natural polymer polyhydroxybutyrate resulting from coalescence of several spherulites grown under temperature jumps is shown. Each spherulite shows occasional rings in the inner region. Tiles of the final solid structure have different forms and sizes, and coalescence of two adjacent spherulites may generate a curve (such as hyperbolas appearing in Fig. 5) at the interface.

Assuming that spherulites originate from their respective centres and, once formed, continue to grow circularly

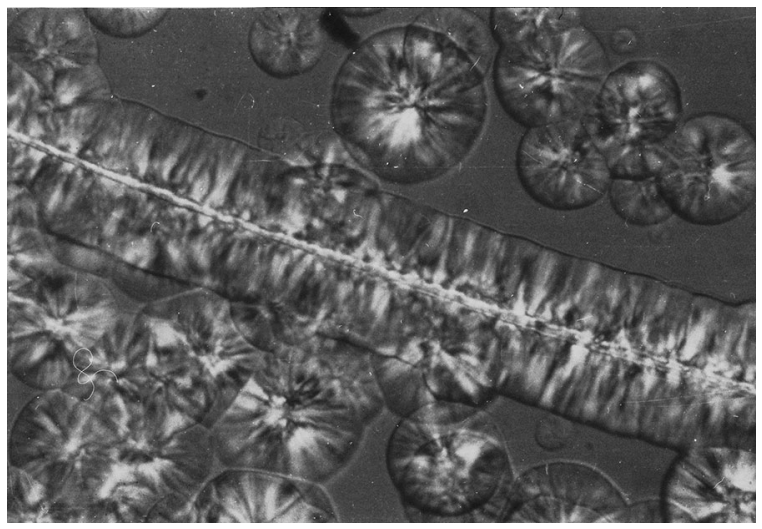

Fig. 4 Polypropylene crystallizing in the presence of a fiber under nominally isothermal conditions

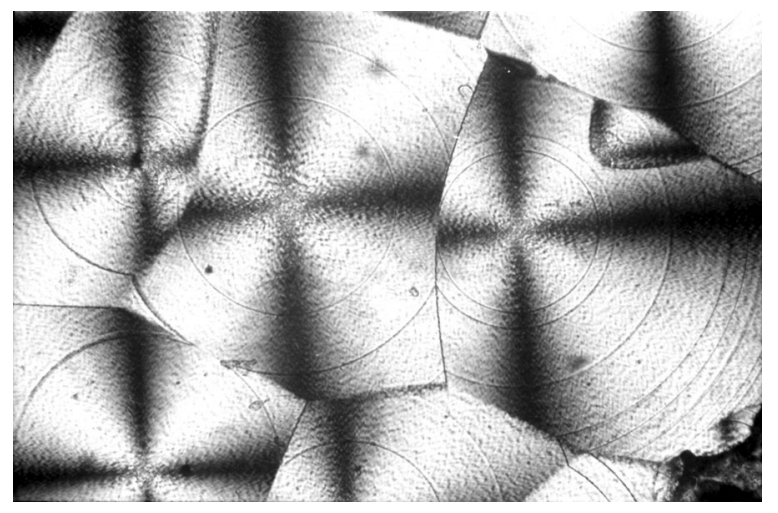

Fig. 5 Coalesced spherulites of polyhydroxybutyrate crystallized under temperature jumps

on their outer edges with the same linear rate as defined before, bigger spherulites must be originated earlier than smaller ones. Moreover, since circles having identical dimensions and linear growth rates must originate lines at the impinged interfaces [19], the presence of curved interfaces in a tessellated structure is a further evidence of a time-dependent nucleation process. Besides information on the dynamics of nucleation and growth, the observation of polymer solids can provide information on the heat flow pattern during cooling from melt. As said before, solidification is exothermal and, therefore, to avoid re-dissolution of crystalline nuclei or stop of growth of their fronts, the developing heat must be rapidly exchanged with the surrounding. In other words, heat has to be eliminated as it is produced at the growth front to allow further solidification. Living organisms adopt different mechanisms to keep constant the body's temperature. For instance, dogs snuggle when it is cold and pant when it is hot. The growth of circular crystalline aggregates, such as spherulites, entails that the medium from which they develop is isotropic with respect to heat propagation that is the rate of heat propagation does not depends on the direction. Isotropic growth of spherulites allows polymer films to offer the maximum solid surface for heat exchange, simultaneously bearing the minimum surface tension. Indeed, compared with other planar geometrical figures with the same "exchanging" area, circles have the smallest boundary and, therefore, the minimum surface energy. In conclusion, spherulites arise, under isotropic conditions along all growth directions, because of their capability to minimize the energy of polycrystalline solids.

\section{Spherulite growth and coalescence}

The solidification of a bi-dimensional polymer sample, according to the theory, occurs by nucleation at different points of the melt and subsequent growth of effective 
nuclei by addition of fresh solid layers to the growth fronts. Liquid-solid transitions in polymers originate a number of circular spherulites (one for each successful nucleation event), whose growth can be observed over the time through an optical microscope. Usually, nucleation is instantaneous, that is all nuclei are formed simultaneously, and all growing spherulites show the same linear rate $\mathrm{d} r / \mathrm{d} t$ and the same radius $r$ instant by instant. However, especially when crystallization occurs by continuous cooling from melt, the number of effective nuclei may be not constant but time dependent. In this case, the linear growth rate is not constant, since it increases with the undercooling $[8,33]$. In any case, when two or more spherulites collide, there are portions of their boundaries unable to growth further, whereas portions still in contact with the liquid phase can continue to grow almost undisturbed. Impingement breaks the circular symmetry of grains and leads to the formation of interfaces. Coalescence of spherulites also causes a gradual decrement (more or less rapid according to the fraction of the coalesced spherulites) of the mass of solid formed per unit of time, i.e., of the true rate of the phase transformation. It is worth to observe that, for a hypothetical monocrystalline polymer specimen with a constant linear growth rate, the crystallization rate (expressed as mass or volume of solids per-unit time) will first increase continuously with more and more rapidity as the circumference of the spherulite increases, and then suddenly fall to zero, together to the drop of the mass of the melt. For real polycrystalline solids, the growth rate first increases, then the increment of the growth rate begins to decrease and finally, briefly after coalescence, the growth rate goes down to zero [12].

For the analysis of growth, let us first focus on a polymer specimen hypothetically crystallizing by simple development of a unique spherulite with a constant linear growth rate $\mathrm{d} r / \mathrm{d} t=g$. By choosing as zero time the nucleation time of the spherulite, the radius of the spherulite at any time $t$ is given by the equation: $r=g t$. Although the spherulite retains its circular form meanwhile it grows continuously by the addition of fresh materials to the boundary, the ratio between the radii of the spherulite at two arbitrary times during crystallization is not constant but depends on the two chosen times: $r_{1} / r_{2}=t_{1} / t_{2}$. The area $A$ of the spherulite is given by the formula: $A=\pi r^{2}=\pi g^{2} t^{2}$, and therefore, it is a function of the square of the time, whereas its derivative (proportional to the whole growth rate) is a linear function of time: $\mathrm{d} A / \mathrm{d} t=2 \pi g^{2} t$. In conclusion, the true growth of a spherulite, showing a constant linear growth rate, depends on the square of time; consequently, the true growth rate is not constant but depends on time. As the surface of a spherulite is proportional to the square of the radius, the ratio
$A\left(t_{1}\right) / A\left(t_{2}\right)=r_{1}^{2} / r_{2}^{2}$ between the total areas of a growing spherulite at two instants $t_{1}$ and $t_{2}$ is also dependent on the choice of the time instants. Indeed, by choosing constant time intervals, that is:

$$
\begin{aligned}
t_{1}-t_{0} & =t_{2}-t_{1}=t_{3}-t_{2}=t_{4}-t_{3}=t_{5}-t_{4} \\
& =\ldots=t_{i+1}-t_{i}=\ldots
\end{aligned}
$$

so that the measurements of the radius at time $t_{1}, t_{2}, t_{3}, t_{4}, t_{5}, \ldots t_{i}, \ldots$ will be:

$r_{1}, 2 r_{1}, 3 r_{1}, 4 r_{1}, 5 r_{1} \ldots, i r_{1}, \ldots$

the ratios between two subsequent areas of a spherulite will result: $4,9 / 4,16 / 9,25 / 16, \ldots(i+1)^{2} /(i)^{2}$.

The above succession converges to 1 for $i \rightarrow \infty$; therefore, the ratio between consecutive areas of a spherulite, achieved after constant time lapses, can be considered constant only for a very long observation time. For a hypothetical monocrystalline sample, coalescence cannot take place and the unique spherulite will grow until liquid phase is still available.

For polycrystalline samples with finite dimensions, the observation time of separate spherulites is limited by impingement, and therefore, true spherulites likely do not achieve the dimensions needed for convergence of the above numerical succession.

Let us estimate the time dependence of the radius increase $\Delta r=r_{i}-r_{i-1}$ of a spherulite in correspondence of two subsequent $i-1$ and $i$ observations. When observations are taken at constant time intervals $\Delta t$, so that: $t_{i}-t_{i-1}=t_{i+1}-t_{i}$, also the increase of the radius $\Delta r=$ $r_{i}-r_{i-1}=r_{i+1}-r_{i}$ at any successive time is constant during the whole crystallization because of the general constancy of linear growth rate. However, as seen above, increments of the area of the spherulite (corresponding to the area of circular coronas) calculated at different crystallization advancements depend not only on the time lapse but also on the difference between the squares of the linear dimensions (i.e., the radii) of the spherulite: $A=\pi\left(r_{i}^{2}-r_{i-1}^{2}\right) \neq \pi(\Delta r)^{2}$. We can conclude that different from the radius, the area of a spherulite does not increase uniformly with time, i.e., the increment of the area of a spherulite is not constant at constant time intervals. This is geometrically evident from the comparison between the areas of two annuli with equal thickness but surrounded by two circumferences with different sizes [12, 32]. Figure 2, for instance, can also be considered as showing the growth of a unique spherulite after two different $\Delta t$ time intervals with equal lengths: the annulus at advanced crystallization is larger than that early grown. Indeed, as the radius of a spherulite increases with a null "acceleration", the area increases with a positive "acceleration" given by the 
formula: $\mathrm{d}^{2} A / \mathrm{d} t^{2}=2 \pi g^{2}$. Since the third derivative $\mathrm{d}^{3} A / \mathrm{d} t^{3}$ of the function $A(t)$ is equal to zero at every time, the first $\mathrm{d} A / \mathrm{d} t$ and the third $\mathrm{d}^{3} A / \mathrm{d} t^{3}$ derivatives of $A(t)$ are each other in the same relationship as that existing between the functions $r(t)$ and $\mathrm{d}^{2} r / \mathrm{d} t^{2}$. Therefore, the analogous of the function $r(t)$ is the first derivative $A^{\prime}(t)=\mathrm{d} A / \mathrm{d} t$ of the function $A(t)$. The derivative $A^{\prime}(t)$ increases uniformly with time generating increments $\Delta A^{\prime}$ constants at constants time intervals, i.e., $A^{\prime}\left(t_{i}\right)-A^{\prime}\left(t_{j}\right)=A^{\prime}\left(t_{h}\right)-A^{\prime}\left(t_{k}\right)$ for $t_{i}-t_{j}=t_{h}-t_{k}$, at least when a single spherulite is monitored in the absence of impingement. Since for a "monocrystalline" polymer film, the function representing the area of the spherulite as a function of time is a stretch of a parabola, also the mass of a growing solid phase as a function of time (i.e., the growth of the spherulite) is represented by a parabola. When multiple nucleation events occur simultaneously, indicating with $n$ the number of spherulites, the total area of the spherulites at any time before impingement will be equal to $n$ times the area of a single spherulite. In this case, depending on the distance between adjacent spherulites, early or late coalescence will occur and the area of the solid will be not more dependent on that of round-shaped crystallites but on the area of progressively decreasing portions of spherulites. Therefore, the mass of a polycrystalline solid increases rapidly with time, as the dimension of spherulites increases before impingement, then the rate of increase begins to decline due to advancement of impingement, finally falling to zero. Namely, the rate of crystallization begins to decrease as coalescence prevails over the portions of spherulite boundaries not yet in contact, achieving the null value when the whole liquid has been transformed in solid. If nucleation is time dependent, the increase of the crystallization rate before impingement is even higher than that found in the case of instantaneous nucleation, since both spherulites size and number increase, i.e., either early spherulites and new appearing spherulites contribute to crystallization growth. However, it is worth noting that if the final number of spherulites is the same in two specimens showing different nucleation types but equal linear growth rates and induction times (i.e., the time needed for crystallization to start), the instantaneously nucleated specimen will also show the higher values of the true growth rate.

Specimens continuously cooled show generally a high crystallization rate just because of the high contribution to the growth rate of premature spherulites, since small spherulites originated later can contribute only marginally to a crystallization rate enhancement. Indeed, during nonisothermal crystallization, $r(t)$ is not linear and the growth rate $\mathrm{d} r / \mathrm{d} t$, as well as the true growth rate $\mathrm{d} m / \mathrm{d} t$, increase remarkably with decreasing temperature. Moreover, the qualitative trend of the crystallization rates is the same for both the cases of instantaneous and non-instantaneous nucleation: soon after the start of impingement, the crystallization rate begins to slowdown. The volume fractions of crystallizing samples plotted against time give generally sigmoid curves. When the crystallization heat develops as a bump-shaped function, the derived curve representing the mass fraction of a polycrystalline solid as a function of time also have a sigmoidal shape [12] and may be used for the kinetic analysis of the phase transition according to Avrami [33-35]. Indeed, integration transforms bumpshaped curves in sigmoid curves. Sigmoid functions are usually "linearized" using the logarithm of the dependent variable. It is worth noting that the crystallization rate is maximum in correspondence of the inflection point of the sigmoid, i.e., when the total length of the lost growth front due to impingement is equal to the length of the still advancing growth fronts of all spherulites.

These intuitive conclusions on the speed of crystallization and on the conditions for isometric growth of spherulites can be mathematically derived as explained in the "Appendixes 1 and 2", respectively, for the case of constant and variable linear growth rates.

\section{Growth of filamentous fungi}

The basic structure of filamentous fungi is referred to as hypha, a tubular filament that originates from a spore [36]. As a hypha grows, it repeatedly branches giving rise to a mass of filaments known as mycelium. For instance, in Fig. 6, the mycelium of a strain of Stemphylium sp. during division is shown.

The knowledge of the rates of production of bacteria and fungi during in situ decomposition processes is very important not only for environmental analysis and

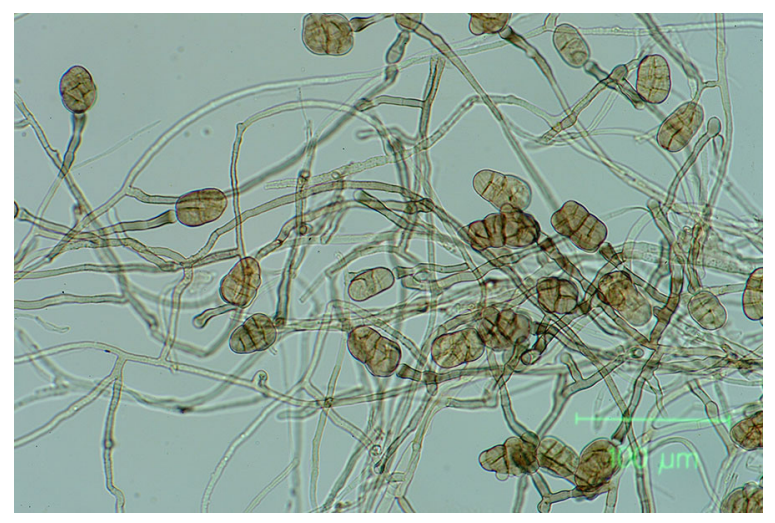

Fig. 6 Optical micrograph showing brown conidia on conidiophores formed during the division of a Stemphylium sp. mycelium. The fungus was grown on a PDA (potato dextrose agar) substrate. Courteously provided by CREA-PAV Plant Pathology Research Centre (Rome) 
protection [37], but also because the microorganism behaviours under different physico-chemical conditions apply to a wide range of disciplines and medical science [38]. Growth can be defined in several ways, such as increase in dry weight, increase in colony radius and rate of production of spores [39], according to the experimental criteria and procedures [11]. When the fungal growth is described by the cumulative weight of the biomass as function of time, under typical in vitro conditions of fixed and large amounts of nutrients, a sigmoidal curve is obtained. There may be a delay before growth starts [40] with a lag time just after $t=0$ (to go deeply into the glucose concentration and water activity dependences of the germination rate see Refs. [22, 40]) followed by an exponential growth and then by a stationary stage. Therefore, a fungal growth curve can be described by three parameters: the lag time, defined as the $x$-axis intercept of the curve; the maximum growth rate, defined as the slope of the tangent in the inflection point and the asymptote, representing the maximum value of growth.

Examples of quantities that follow such growth curves in living matter are the length or mass of a human, a potato, or a fish and the extent of a population of fish or microorganisms [38]. Exponential growth is observed not only by measuring the fungal mass in submerged cultures, but also estimating the length (whose logarithm is proportional to time) of germ tubes grown on solid substrates $[11,41]$. When the growth is followed by measuring the radial extension of Petri plate inoculum as a function of time, data are generally fitted by lines, that is the growth is linear and the linear growth rate is constant. As explained below, a constant linear growth rate of a circular mycelium originated in the center of a Petri dish conforms with an exponential, and not linear, growth of the dry mass. Indeed, the depletion of nutrients inside the colony and their high availability at the growth front has led to the concept of a peripheral annulus, where both the increases of hyphal length and of the number of hypha tips originated by branching produce an exponential increase of the fungal mass. In other words, like polymer spherulites, rounded fungal growth may be considered to occur in outer shells surrounding an inner mass of non-growing mycelium. The wideness of the peripheral growth zone depends on the particular fungus, and usually, in septate fungi, it is considerably shorter than in non-septate fungi [42]. For instance, the length of the peripheral zone for Penicillium chrysogenum is $496 \mu \mathrm{m}$, whereas for Mucor racemosus is only $3.41 \mu \mathrm{m}[21,42]$. For some fungi, the cube root, rather than logarithm, of the dry weight may be found proportional to time, especially in the final phase of a long growth experiment. This kinetic change is ascribed to the aggregation of mycelium in tight pellets, limiting the movement of nutrients and oxygen within the pellets and thus narrowing growth of hyphae and branches only at a very thin, exterior layer of the pellets.

Fungal development is affected by nutritional necessities and environmental availability. For instance, the ratio $\mathrm{C}: \mathrm{N}$ in the growth medium, as well as the presence of some minerals, are fundamental to sustain an appreciable fungal growth [37, 43, 44]. On the other side, environmental factors, such as water activity, temperature, ph and oxygen availability, or the presence of inhibitors and waste products, may strongly affect the fungal growth rate. During growth, changes in several quantities and, hence, in growth rates, occur: nutrients diminish and waste products accumulate, often accompanied by changes in $\mathrm{pH}$ and $\mathrm{O}_{2}$ availability. For easy interpretation of kinetics results, it is necessary to make most of the parameters constant during growth, varying one factor at once. It is also needed to select the culture method most appropriate to the objective of the growth experiment. For instance, it has been shown that the germ-tube method is inappropriate to study the effect of nutrients upon growth [21].

As discussed above, when an axenic strain of a filamentous fungus is inoculated in a Petri dish, the fungal growth can be followed by measuring the diameter of the colony, instead of the dry mass [39]. This is the most suitable method to highlight the analogies between the development of fungi and polymer solids. Indeed, as fibrillar crystals forming polymer spherulites, hyphae elongate apically also developing lateral branches, which, once formed, grow themselves at the tips. As shown by Trinci [21], the radial growth rates of circular fungal colonies $K_{r}$, equivalent to the linear growth rates of spherulites, are found to be constant under specific nutritional and surrounding conditions. In other words, the radius of fungal colonies, coinciding with the length of leading hyphae at the peripheral growth zone, increases linearly with time according to the equation:

$R=R_{0}+K_{r}\left(t-t_{0}\right)$,

where $R$ is the colony radius or hyphal length at time $t, R_{0}$ is the colony radius or hyphal length at $t_{0}$, and $K_{r}$ is the constant linear growth rate. Since branching does not contribute to the radial development of the colony but contributes to the increment of the fungal mass, the true growth of the fungus is not linear but exponential.

In submerged shake-flask culture, indeed, the mass of filamentous fungi is found to grow exponentially according to the equation:

$\frac{\mathrm{d} M}{\mathrm{~d} t}=\alpha M$,

where $M$ is the mass of culture at time $t$ and $\alpha$ is a constant, at a prefixed temperature, known as the "specific growth rate" (i.e., the growth rate of a unitary mass of culture perunit time). Integration of the above equation gives: 
$\ln \frac{M}{M_{0}}=\alpha t$

where $M_{0}$ is the mass of organism at an arbitrary time. The specific growth rate $\alpha$ of an organism may be, therefore, calculated from the expression:

$\alpha=\ln 2 / t_{\mathrm{d}}$

where $t_{\mathrm{d}}$ is the doubling or generation time, which represents the time needed to have an increase of $100 \%$ of the original mass $M_{0}$ of the organism (i.e., so that $M=2 M_{0}$ ). This means that if the growth of a colony depends exponentially on the time (i.e., $M=M_{0} \mathrm{e}^{\alpha t}$ ) and the doubling time is constant, the specific growth rate $\alpha$ can be related to the theoretically radial growth rate $K_{r t}$ through the formula $[21,22]$ :

$K_{r t}=\alpha w$,

where $w$ is the experimentally determined width of the colony's peripheral growth zone in $\mu \mathrm{m}$.

The two different fungal growth functions, namely, linear with respect to the radial and exponential relatively to the area/volume extensions, are explainable by the mechanism of hyphal branching. Indeed, only growth of preexisting hyphae contributes to the radial development; meanwhile, the increase of the hyphal density due to branching in the peripheral growth zone does not contribute to the colony's radial expansion [21, 22]. Moreover, the contribution of branching to the fungal mass increases with increasing the radius of the colony, because later branches may undergo further subdivision. This continuous process of subdivision of lateral branches of hyphae is evidently responsible for the exponential increase of the true growth of fungi.

\section{Conclusions}

The growth mechanisms of polymer spherulites have been described and discussed in comparison with growth of biological systems. It has been shown that metric considerations allow to correlate kinetics with morphology of growing systems and their possible interfaces.

The growth mechanism of filamentous fungi under homogeneous and unrestricted conditions can be compared to that of polymer spherulites. As several polymer spherulites, circular mycelia grow in the peripheral zone by concurrent elongation and branching of hyphae, since the local accumulation of waste products and rhythmic depletion of nutrients at the hyphal apex during linear extension stimulate branching in directions. where a high concentration of food is still available. Indeed, branches are found to extend first quickly than the parents hyphae, then the linear growth rate of branches decreases, eventually falling to the value of the hyphae from which they are derived. Because of heat development during liquid-solid transformations, a similar growth mechanism of fibrillar crystals in polymer spherulites takes place owing to heat accumulation at the tip of radial crystals and the possibility for crystals to elongate through branching in non-radial directions, where local lower temperatures favour a faster heat removal. Spirally grown spherulites may grow even faster than branched spherulites; because their effective growth front is larger and heat may be released easily before enfolding with new solid material.

Acknowledgments The present authors would like to thank CREAPAV Plant Pathology Research Centre (Rome) for kindly providing the picture of Stemphylium sp.

\section{Appendix 1: Growth of spherulites with constant linear rates}

It is intuitive that spherulites, simultaneously nucleated and growing with the same linear growth rate, have also equal dimensions and growth rates at any time. The growth of a couple of such spherulites is, therefore, isometric for the whole crystallization, at least before impingement with neighbours takes place. We will also see that, when two spherulites do not nucleate simultaneously, isometric grow is not possible at all, independently of the linear growth rate values. Moreover, we will establish that simultaneous nucleation is a necessary and sufficient condition to ensure isometry, even when the two spherulites grow with different, but constant, linear rates. It is worth noting that to assess if growth is or not isometric, it is necessary to estimate the ratio between the areas of two spherulites at different times. However, for predictions on the metrics of growth, the ratio between the areas can be conveniently replaced by the ratio between the radii of growing spherulites. Indeed, the constancy of the ratio $\left(r_{1} / r_{2}\right)^{2}$ between the areas of two spherulites entails the constancy of the ratio $r_{1} / r_{2}$ between the radii of the spherulites, and vice versa.

Considering two growing spherulites 1 and 2 having respective radii $r_{1}, r_{1}^{\prime}$ and $r_{2}, r_{2}^{\prime}$ at two instants $t$ and $t^{\prime}$, to observe isometric growths at two consecutive observations it must be: $r_{1} / r_{2}=r_{1}^{\prime} / r_{2}^{\prime}$, i.e., $r_{1} r_{2}^{\prime}=r_{1}^{\prime} r_{2}$ or $r_{1} / r_{1}^{\prime}=r_{2} / r_{2}^{\prime}$. This condition is always valid for instantaneous spherulites growing with equal growth rates, since for the most part of the growth (i.e., up to impingement), it results: $r_{1}=r_{2}$ and $r_{1}^{\prime}=r_{2}^{\prime}$.

To analyse the general case of different nucleation times and growth rates, let us observe that the sum and the product of the radius $r$ and $r^{\prime}$ of an expanding circle at two instants $t$ and $t^{\prime}$ are coefficients of one of the set of parabolas with equation: 
$y=2 x^{2}-2\left(r+r^{\prime}\right) x+2 r r^{\prime}$.

The symmetry axes of such parabolas are the vertical lines with equation: $x=\left(r+r^{\prime}\right) / 2$, whereas the vertexes are placed in the points: $\left(\left(r+r^{\prime}\right) / 2,2 r r^{\prime}-\left(r+r^{\prime}\right)^{2} / 2\right)$. Hence, the lengths of the radius of a spherulite at two successive instants can be obtained as solutions of the equation:

$x^{2}-\left(r+r^{\prime}\right) x+r r^{\prime}=0$.

Since the radii of two spherulites nucleated simultaneously and growing with constant linear rates can be, respectively, described by the equations:

$y=2 x^{2}-2\left(r_{1}+r_{1}^{\prime}\right) x+2 r_{1} r_{1}^{\prime}$

$y=2 x^{2}-2\left(r_{2}+r_{2}^{\prime}\right) x+2 r_{2} r_{2}^{\prime}$

to obtain the radii of the two spherulites at the two instants $t$ and $t^{\prime}$ it is sufficient to solve the two systems:

$\left\{\begin{array}{l}y=2 x^{2}-2\left(r_{1}+r_{1}^{\prime}\right) x+2 r_{1} r_{1}^{\prime} \\ y=0\end{array}\right.$
$\left\{\begin{array}{l}y=2 x^{2}-2\left(r_{2}+r_{2}^{\prime}\right) x+2 r_{2} r_{2}^{\prime} \\ y=0\end{array}\right.$

or, equivalently, to solve the two equations:

$x^{2}-\left(r_{1}+r_{1}^{\prime}\right) x+r_{1} r_{1}^{\prime}=0$

$x^{2}-\left(r_{2}+r_{2}^{\prime}\right) x+r_{2} r_{2}^{\prime}=0$.

For two spherulites nucleated simultaneously and growing with equal constant rates, it results instant by instant: $r_{1}=r_{2}$ and $r_{1}^{\prime}=r_{2}^{\prime}$; therefore, the radii are also solutions of the systems:

$\left\{\begin{array}{l}y=2 x^{2}-2\left(r_{1}+r_{2}^{\prime}\right) x+2 r_{1} r_{2}^{\prime} \\ y=0\end{array}\right.$

$\left\{\begin{array}{l}y=2 x^{2}-2\left(r_{2}+r_{1}^{\prime}\right) x+2 r_{2} r_{1}^{\prime} \\ y=0\end{array}\right.$

i.e., the radii are also solutions of the equations:

$x^{2}-\left(r_{1}+r_{2}^{\prime}\right) x+r_{1} r_{2}^{\prime}=0$

$x^{2}-\left(r_{1}^{\prime}+r_{2}\right) x+r_{1}^{\prime} r_{2}=0$.

Indeed, if two spherulites appear simultaneously and grow with the same rate (i.e. $r_{1}=r_{2}$ and $r_{1}^{\prime}=r_{2}^{\prime}$ ) the two equations:

$y=x^{2}-\left(r_{1}+r_{2}^{\prime}\right) x+r_{1} r_{2}^{\prime}$

$y=x^{2}-\left(r_{1}^{\prime}+r_{2}\right) x+r_{1}^{\prime} r_{2}$

represent the same parabola and, therefore, the two systems: $\left\{\begin{array}{l}y=x^{2}-\left(r_{1}+r_{2}^{\prime}\right) x+r_{1} r_{2}^{\prime} \\ y=0\end{array} ;\left\{\begin{array}{l}y=x^{2}-\left(r_{1}^{\prime}+r_{2}\right) x+r_{1}^{\prime} r_{2} \\ y=0\end{array}\right.\right.$

admit the same solutions $\left(r_{1}=r_{2}, 0\right),\left(r_{1}^{\prime}=r_{2}^{\prime}, 0\right)$ for any couple of observations $\left(t, t^{\prime}\right)$. This demonstrates that two simultaneously nucleated spherulites growing with equal rates have equal ratio between radii at any time and, therefore, grow isometrically.

However, the condition of equal linear growth rates is not necessary to have isometric growth when two spherulites nucleate simultaneously. Indeed, for any couple of constant linear rates $g_{1}$ and $g_{2}$ relative to two instantaneous spherulites, it is possible to choose as zero time the unique nucleation instant $t_{1}^{0}=t_{2}^{0}=0$, so that it results: $r_{1} / r_{1}^{\prime}=$ $g_{1} t / g_{1} t^{\prime}=t / t^{\prime}$ and $r_{2} / r_{2}^{\prime}=g_{2} t / g_{2} t^{\prime}=t / t^{\prime}$. This also entails that for any couple of times $\left(t, t^{\prime}\right)$, it results $r_{1} / r_{2}=r_{1}^{\prime} / r_{2}^{\prime}$, i.e., the growth is isometric at any time, at least up to impingement.

Vice versa, for two non-simultaneously nucleated spherulites, $t_{1}^{0} \neq t_{2}^{0}$, and therefore, it results also:

$$
r_{1} / r_{2}^{\prime}=g_{1}\left(t-t_{1}^{0}\right) / g_{1}\left(t^{\prime}-t_{1}^{0}\right) \neq r_{2} / r_{2}^{\prime}=g_{2}\left(t-t_{2}^{0}\right) / g_{2}\left(t^{\prime}-t_{2}^{0}\right),
$$

i.e., crystallization cannot be isometric, independently of the exact values of $g_{1}$ and $g_{2}$.

Briefly, we can say that simultaneous nucleation is a sufficient condition for isometric growth of spherulites; assuming an instantaneous nucleation, the growth of a couple of separate spherulites is isometric up to impingement at any observation time, either if spherulites grow with the equal or different linear rates. Indeed, for a spherulite growing with linear rate $g$, the ratio between two subsequent measures of the radius is independent of $g$ :

$\frac{r}{r^{\prime}}=\frac{g t}{g t^{\prime}}=\frac{t}{t^{\prime}}$.

Hence, for any couple of simultaneous spherulites considered at two instants $t$ and $t^{\prime}$ is verified the condition for isometric growth:

$\frac{r_{1}}{r_{1}^{\prime}}=\frac{r_{2}}{r_{2}^{\prime}}$.

The growth of two separate spherulites not instantaneously nucleated is instead generally allometric during crystallization, since the above condition can never be satisfied whatever be the values of the constant linear rates. Indeed, assuming that $n$ spherulites, nucleated at different times during crystallization, grow with constant linear rates $g_{n}$, the radius $r_{n}$ of each spherulite as a function of time will be given by:

$$
\begin{aligned}
& r_{1}=g_{1}\left(t-t_{1}^{0}\right) ; \\
& r_{2}=g_{2}\left(t-t_{2}^{0}\right) ; r_{3}=g_{3}\left(t-t_{3}^{0}\right) ; \ldots r_{n}=g_{n}\left(t-t_{n}^{0}\right) .
\end{aligned}
$$


In (1) $t_{1}^{0}, t_{2}^{0}, t_{3}^{0}, \ldots t_{n}^{0}$ are, respectively, the times elapsed for nucleation of each spherulite, theoretically measured from an arbitrary zero time, and each of Eq. (1) has physical meaning (i.e., positive radius) only for $t>t_{i}^{0}$. Although $t_{1}^{0}, t_{2}^{0}, t_{3}^{0}, \ldots t_{n}^{0}$ are not directly measurable, they can be extrapolated from $r-t$ lines for $r \rightarrow 0$.

From (1), it follows that for any couple $p, q$ of spherulites considered at two times $t$ and $t^{\prime}$ :

$$
\begin{aligned}
& r_{p} / r_{q}=g_{p}\left(t-t_{p}^{0}\right) / g_{q}\left(t-t_{q}^{0}\right) \quad \text { for } \quad p, q=1,2,3, \ldots n \\
& r_{p}^{\prime} / r_{q}^{\prime}=g_{p}\left(t^{\prime}-t_{p}^{0}\right) / g_{q}\left(t^{\prime}-t_{q}^{0}\right) \text { for } p, q=1,2,3, \ldots n
\end{aligned}
$$

and, therefore, the ratios $r_{p} / r_{q}$ is constant (and equal to $\left.g_{p} / g_{q}\right)$ only if $t_{p}^{0}=t_{q}^{0}$. The condition of constancy of the ratio $r_{p} / r_{q}$ can be only ensured by two spherulites simultaneously nucleated; for simultaneous spherulites growing with the same linear rate $g$, it results $r_{p}=r_{q}$ for the whole crystallization and, hence, spherulites are identical at any time.

In conclusion, a unique constant value of the linear growth rate is not sufficient to ensure isometric growth of spherulites, whereas two instantaneous spherulites grow always isometrically, independently of their exact linear growth rate values.

\section{Appendix 2: Time- and space-dependent linear growth rates}

We will here calculate, at various times during crystallization, the relative dimensions of spherulites sporadically nucleated and growing with non-constant linear rate, in order to assess the conditions under which the ratio between the radii remains constant with time.

In the general case of non-constant, nor uniform, linear growth rates $G(t)$, the functions $r(t)$ are not lines but curves. A sudden heating of the growth front might cause a momentary stop of solidification or even the melting of material at the growth front $[8,12]$, with a temporarily short contraction of spherulites. However, to avoid superheating at the growth front, solidification is generally carried out under suitable temperature programs, which ensure a sudden cooling at a prefixed low temperature (isothermal crystallization) or a progressive cooling at a prefixed rate (non-isothermal crystallization). Since there are not large increases of temperature during regular crystallization procedures, the functions $r(t)$ are strictly monotonically ascending.

For a time-dependent nucleation and non-constant but uniform growth rates $G(t)$, indicating with $r_{n}^{0}$, the radius of the $n$th spherulite at a prefixed zero time and assuming that all spherulites grow instant by instant with the same rate
$G(t)$, independently of the exact type of $G-t$ function the radius of the $n$th spherulite at a generic time $t$ will be given by:

$r_{n}(t)=r_{n}^{0}+\int_{0}^{t} G(t) \mathrm{d} t$.

It follows, as already shown in the "Appendix 1", that two spherulites 1 and 2 characterized by the same constant growth rate function $G(t)$ show an isometric growth, that is a constant $r_{1} / r_{2}$ ratio, only if they nucleated simultaneously, that is if $r_{1}^{0}=r_{2}^{0}$.

More broadly, to have isometric growth of two separated spherulites up to impingement, the two following conditions must be verified: nucleation has to be instantaneous (a condition usually characteristic of isothermal crystallization) and the ratio between the functions $G_{1}(t)$ and $G_{2}(t)$ must be constant, that is $G_{1}(t)=$ const $\cdot G_{2}(t)$ at any time. This latter condition ensures that two simultaneous spherulites mantain proportional sizes for the whole crystallization. Indeed, under both the mentioned conditions, two primitives $r_{1}(t)$ and $r_{2}(t)$ of the functions $G_{1}(t)$ and $G_{2}(t)$ exists so that $r_{1}(t) / r_{2}(t)=$ const.

In conclusion, isometric growth of two (or more) spherulites is possible only if spherulites nucleate simultaneously and the relative $r-t$ curves (or lines in the case of constancy of the linear growth rates) may be obtained one from another by a contraction or dilatation along the ordinate axis. This means that the $r-t$ curves of two spherulites intersect the abscisse axis in the same point and the graph of a spherulite radius can be obtained by moving all points, relative to a smaller spherulite, away from the $t$ axis to a multiple of their original distance from the axis. Numerically, this vertical dilatation entails that the radius of the larger spherulite at any time is obtained multiplying that of the smaller spherulite by a same coefficient $c>1$ (see Fig. 7). Therefore, in the case of non-constancy of the linear growth rates, instantaneous nucleation is a necessary but not sufficient condition for isometric growth of spherulites.

It is worth noting that if the ratio between the radii of two separate $P$ and $Q$ spherulites remains constant during solidification, also the ratio between the spherulite areas $\left(r_{P} / r_{Q}\right)^{2}$ is constant with time and it remains constant even if the two spherulites impinge each other, but no coalescence occurs with anyone of the other $n-2$ spherulites. If the impingement of all groups of spherulites is considered to occur simultaneously and uniformly in space and the distances amongst the centers of adjacent spherulites are the same in all coalesced particles, these latter have identical shape and dimension instant by instant. In such an ideal system, coalesced solid particles are constituted of the same number of spherulites and their relative growth remains isometric up to complete crystallization. 


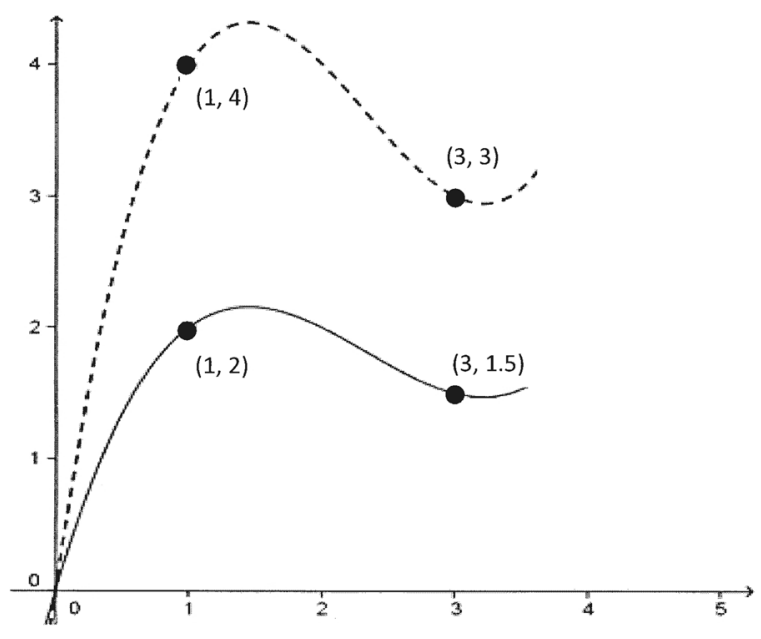

Fig. 7 Example of two functions linked by the relationship: $f_{1}(x)=c f_{2}(x)$. The values of one function may be obtained from those of the other function through the coefficient $c$

When two spherulites $S$ and $Z$ grow with the same linear rate, i.e., $G_{S}(t)=G_{Z}(t)$, but with different dimensions due to their different nucleation times, their surface increments in a certain time interval $\Delta t=t^{\prime}-t$ will be given respectively by:

$2 \pi\left(r_{S}^{\prime 2}-r_{S}^{2}\right)=2 \pi\left(r_{S}^{\prime}-r_{S}\right)\left(r_{S}^{\prime}+r_{S}\right)$

$2 \pi\left(r_{Z}^{\prime 2}-r_{Z}^{2}\right)=2 \pi\left(r_{Z}^{\prime}-r_{Z}\right)\left(r_{Z}^{\prime}+r_{Z}\right)$.

Since $\left(r_{S}^{\prime}-r_{S}\right)=\left(r_{Z}^{\prime}-r_{Z}\right)$, the relative surface increase of the two spherulites is equal to the ratio between the sum of the radii of each spherulite at the time $t^{\prime}$ and $t$ :

$2 \pi\left(r_{S}^{\prime 2}-r_{S}^{2}\right) / 2 \pi\left(r_{Z}^{\prime 2}-r_{Z}^{2}\right)=\left(r_{S}^{\prime}+r_{S}\right) /\left(r_{Z}^{\prime}+r_{Z}\right)$.

Defining $g$ as the average linear growth rate of each spherulite in the interval $\Delta t$, the above ratio can be rewritten as:

$\left(r_{S}^{\prime}+r_{S}\right) /\left(r_{Z}^{\prime}+r_{Z}\right)=\left(2 r_{S}+g \Delta t\right) /\left(2 r_{Z}+g \Delta t\right)$.

If $r_{S} \neq r_{Z}$, i.e., if the two spherulites are not simultaneous, the above ratio cannot be constant within the interval $\Delta t$.

Analogously, we can say that the ratio $\left(r_{S} / r_{Z}\right)^{2}$ between the areas of the two spherulites at a certain time $t$ :

$\left[r_{s}^{0}+g\left(t-t_{s}^{0}\right)\right]^{2} /\left[r_{z}^{0}+g\left(t-t_{z}^{0}\right)\right]^{2}$

cannot be constant with time if $t_{S}^{0} \neq t_{Z}^{0}$.

It also follows that two spherulites not simultaneously nucleated will begin to grow allometrically (with a positive growth if $r_{S} / r_{Z}>1$, negative if $r_{S} / r_{Z}<1$ ), whatever the growth rate function be. Although spherulites not simultaneously nucleated and growing with equal instantaneous rates maintain, before impingement, their relative dimensions in the sense that the early spherulite will be always larger than the late spherulite, the ratio between dimensions of such spherulites cannot remain constant during crystallization.

Furthermore, if a number of spherulites grow at different rates, the size of later spherulites can even overcome that of a spherulite nucleated earlier but growing slowly. Under this circumstance, in correspondence of the time $t_{e}$ needed for achievement of identical size, the allometric growth of a couple of spherulites $S$ and $Z$ might exhibit a change of the sign or even, under the conditions $r_{S}\left(t_{e}\right)=r_{Z}\left(t_{e}\right)$ and $G_{S}(t)=$ const $G_{Z}(t)$ for $t>t_{e}$, a change towards isometric growth for $t>t_{e}$.

In conclusion, if the growth rate functions of two spherulites are not constant with time, isometric growth up to impingement is not possible unless the two spherulites nucleate simultaneously, and the ratio between their growth rate functions is constant. In other words, isometric growth in isothermal crystallization of polymers would be an exception, rather than the norm, if the growth rate functions were not constant. If the linear growth rates of two spherulites are both constant with time, the condition of constancy of their ratio is also verified and therefore it is sufficient simultaneous nucleation to ensure isometry.

\section{References}

1. Tojo B, Ohno T (1999) Continuous growth-line sequences in gastropod shells. Paleogeogr Paleoclimatol Paleoecol 145:183-191

2. Jones DS, Arthur MA, Allard DJ (1989) Sclerochronological records of temperature and growth from shells of Mercenaria mercenaria from Narragansett Bay, Rhode Island. Mar Biol 102:225-234

3. Raimo M (2004) Influence of temperature changes on crystallization of an ethylene-propylene random copolymer. J Appl Polym Sci 94:2008-2013

4. Wang Z, An L, Jiang B, Wangh X (1998) Periodic radial growth in ring-banded spherulites of poly( $\varepsilon$-caprolactone)/poly(styreneco-acrylonitrile) blends. Macromol Rapid Commun 19:131-133

5. Kyu T, Chiu H-W, Guenthner AJ, Okabe Y, Saito H, Inoue T (1999) Rhythmic growth of target and spiral spherulites of crystalline polymer blends. Phys Rev Lett 83:2749-2752

6. Bianchi DE (1964) An endogenous circadian rhythm in Neurospora crassa. J Gen Microbiol 35:437-445

7. Bell-Pedersen D (2000) Review. Understanding circadian rhythmicity in Neurospora crassa: from behavior to genes and back again. Fungal Genet Biol 29:1-18

8. Raimo M (2004) Delay in the growth of polymer spherulites caused by superheating. J Mater Sci Lett 39:5567-5568

9. Dunlap JC (1999) Molecular bases for circadian clocks. Cell 96:271-290

10. Douady S, Couder Y (1992) Phyllotaxis as a physical self-organized growth process. Phys Rev Lett 68:2098-2101

11. Griffin DH (1996) Fungal physiology, 2nd edn. Wiley, New York, pp 102-103 
12. Raimo M (2015) Growth of spherulites: foundation of the DSC analysis of solidification. Chemtexts 1:13-33

13. Raimo M (2014) Structure and morphology of polyoxymethylene. In: Sigrid L, Visakh PM, Sarath C (eds) Polyoxymethylene handbook, Chap 6. Scrivener publishing-Wiley, pp 163-191

14. Sigler L (2002) Fibonacci's Liber Abaci. A translation into modern English of Leonardo Pisano's book of calculation, Springer-Verlag New York, Inc

15. Medawar PB (1941) The 'laws' of biological growth. Nature 148:772-774

16. Kent M (2000) Advanced biology. Oxford University Press, Oxford

17. Raimo M (2011) An overview on the processing of polymers growth rate data and on the methods to verify the accuracy of the input parameters in crystallization regime analysis. Progr Cryst Growth Charact Mater 57:65-92

18. Stefan J (1891) On the theory of formation of ice, in particular in the polar sea. Annalen der Physik und Chemie (Wiedermann) 42:269-286

19. Raimo M (2007) "Kinematic" analysis of growth and coalescence of spherulites for predictions on spherulitic morphology and on the crystallization mechanism. Progr Polym Sci 32:597-622

20. Castro-Longoria E, Brody S, Bartnicki-García S (2007) Kinetics of circadian band development in Neurospora crassa. Fungal Genet Biol 44:672-681

21. Trinci APJ (1971) Influence of the width of the peripheral growth zone on the radial growth rate of fungal colonies on solid media. J Gen Microbiol 67:325-344

22. Trinci APJ (1969) A kinetic study of the growth of Aspergillus nidulans and other fungi. J Gen Microbiol 57:11-24

23. Shtukenberg AG, Punin YO, Gunn E, Kahr B (2012) Spherulites. Chem Rev 112:1805-1838

24. Norton DR, Keller A (1985) The spherulitic and lamellar morphology of melt-crystallized isotactic polypropylene. Polymer 26:704-716

25. Gránásy L, Pusztai T, Tegze G, Warren JA, Douglas JF (2005) Growth and form of spherulites. Phys Rev E 72:0116051

26. Burnaby TP (1966) Allometric growth of ammonoid shells: a generalization of the logarithmic spiral. Nature 209:904-906

27. Bennema P (1969) The importance of surface diffusion for crystals growth from solution. J Crys Growth 5:29-43

28. Okabe Y, Kyu T, Saito H, Inoue T (1998) Spiral crystal growth in blends of poly(vinylidene fluoride) and poly(vinyl acetate). Macromolecules 31:5823-5829
29. Smereka P (2000) Spiral crystal growth. Physica D 138:282-301

30. Choy CL (1977) Thermal conductivity of polymers. Polymer 18:984-1004

31. Choy CL, Leung WP (1983) J Polymer Sci Polymer Phys Ed 21:1243-1246

32. Raimo M (2015) On the origin of transcrystalline morphology in polymers and their composites: re-evaluation of different views. Mater Today Commun 3:137-140

33. Avrami M (1941) Granulation, phase change, and microstructure kinetics of phase change. III. J Chem Phys 9:177-184

34. Avrami M (1939) Kinetics of phase change. I General theory. J Chem Phys 7:1103-1112

35. Avrami M (1940) Kinetics of phase change. II Transformationtime relations for random distribution of nuclei. J Chem Phys $8: 212-224$

36. Bartnicki-Garcia S, Girbardt M, Bracker C (2015) Outstanding pioneers in fungal microscopy. Nat Rev Microbiol 13:52-57

37. Rousk J, Bååth E (2007) Fungal and bacterial growth in soil with plant materials of different $\mathrm{C} / \mathrm{N}$ ratios. FEMS Microbiol Ecol 62:258-267

38. Zwietering MH, Jongenburger I, Rombouts FM, Van't Riet K (1990) Modeling of the bacterial growth curve. Appl Environ Microbiol 56:1875-1881

39. Ayerst G (1969) The effects of moisture and temperature on growth and spore germination in some fungi. Food Stored Prod Res 5:127-141

40. Pardo E, Marín S, Solsona A, Sanchis V, Ramos AJ (2004) Modeling of germination and growth of ochratoxigenic isolates of Aspergillus ochraceus as affected by water activity and temperature on a barley-based medium. Food Microbiol 21:267-274

41. Smith JH (1924) On the early growth rate of the individual fungus hypha. New Phytol 2:65-78

42. Finkelstein DB (1992) Transformation. In: Finkelstein DB, Ball C (eds) Biotechnology of Filamentous Fungi-technology and products. Butterworth-Heinemann, Boston

43. Rousk J, Brookes PC, Bååth E (2010) Investigating the mechanisms for the opposing $\mathrm{pH}$ relationships of fungal and bacterial growth in soil. Soil Biol Biochem 42:926-934

44. Marinelli E, Orzali L, Lotti E, Riccioni L (2012) Activity of some essential oils against pathogenic seed borne fungi on legumes. Asian J Plant Pathol. doi:10.3923/ajppaj.2012 